\title{
SEND Version 3.0 Draft A
}

National Cancer Institute

\section{Source}

National Cancer Institute. SEND Version 3.0 Draft A. NCI Thesaurus. Code C90453.

The A draft of the standard for the exchange of non-clinical data (SEND) implementation guide. 